LETTER TO THE EDITOR

\title{
The spectroscopic evolution of the recurrent nova T Pyxidis during its 2011 outburst (Corrigendum)
}

\section{The optically thick phase and the origin of moving lines in novae}

\author{
S. N. Shore ${ }^{1}$, T. Augusteijn ${ }^{2}$, A. Ederoclite ${ }^{3,4}$, and H. Uthas ${ }^{5}$ \\ 1 Dipartimento di Fisica "Enrico Fermi”, Università di Pisa, and INFN- Sezione Pisa, largo B. Pontecorvo 3, 56127 Pisa, Italy \\ e-mail: shore@df.unipi.it \\ 2 Nordic Optical Telescope, Apartado 474, 38700 Santa Cruz de La Palma, Santa Cruz de Tenerife, Spain \\ e-mail: tau@not.iac.es \\ 3 Instituto de Astrofísica de Canarias, 38200, La Laguna, Tenerife, Spain \\ e-mail: ale@iac.es \\ ${ }^{4}$ Departamento de Astrofísica, Universidad de La Laguna, 38206 La Laguna, Tenerife, Spain \\ 5 Department of Astronomy, Columbia University, 550 W 120th Street, New York, NY 10027, USA \\ e-mail: helena@astro.columbia.edu
}

A\&A 533, L8 (2011), DOI: 10.1051/0004-6361/201117721

\section{ABSTRACT}

We correct the velocities quoted for interstellar absorption features from Shore et al. (2011), A\&A, 533, L8.

Key words. novae, cataclysmic variables - stars: individual: T Pyx - errata, addenda

Due to an error in the application of the heliocentric corrections to the NOT radial velocities, the interstellar velocities quoted in the paper (and in Table 2) for the optical lines require corrections of $-19.6 \mathrm{~km} \mathrm{~s}^{-1}$ for the dates covered in the text. Catchpole (1969). Also gives radial velocities for the two components on Ca II K, +19.2 and $+20.6 \mathrm{~km} \mathrm{~s}^{-1}$ based on photographic spectra taken at $49 \AA \mathrm{mm}^{-1}$ during the 1966 outburst. The corrected Table 2 is included here. We thank Ivan De Gennaro Aquino, Univ. di Pisa, whose diligent work uncovered this problem.

\section{References}

Catchpole, R. M. 1969, MNRAS, 142, 119
Table 2. Heliocentric interstellar line velocities.

\begin{tabular}{lccc}
\hline \hline Identification & $\lambda(\AA)$ & Abs/em & $v_{\text {rad }} \mathrm{km} \mathrm{s}^{-1}$ \\
\hline Ca II K & 3933.66 & abs & $14.8,22.7$ \\
$\mathrm{CH}^{+}$ & 4232.54 & abs & 17.8 \\
$\mathrm{CH}$ & 4300.31 & abs & 29.9 \\
$\mathrm{Na}$ I D1 & 5889.95 & abs & $11.1,21.2$ \\
$\mathrm{Na}$ I D2 & 5895.92 & abs & $10.8,22.9$ \\
$\mathrm{H} \mathrm{I}$ & $21 \mathrm{~cm}$ & $\mathrm{em}$ & $11.4,21.9,34.8,81.1$ \\
\hline
\end{tabular}

